\title{
Glucocorticoid Receptor Alpha Targets SLC2A4 to Regulate Protein Synthesis and Breakdown in Porcine Skeletal Muscle Cells
}

\author{
Xiao-Li Du ${ }^{\dagger}$, Wei-Jing Xu ${ }^{\dagger}$, Jia-Li Shi, Kai Guo, Chang-Tong Guo, Rong Zheng, Si-Wen Jiang and Jin Chai * \\ College of Animal Science and Technology, Huazhong Agricultural University, Wuhan 430070, China; \\ 15207131682@163.com (X.-L.D.); 2385685069@163.com (W.-J.X.); realscary@foxmail.com (J.-L.S.); \\ 15038027365@163.com (K.G.); Gct792808035@163.com (C.-T.G.); zhengrong@mail.hzau.edu.cn (R.Z.); \\ jiangsiwen@mail.hzau.edu.cn (S.-W.J.) \\ * Correspondence: chaijin@mail.hzau.edu.cn; Tel.: +86-189-8617-1213 \\ + These authors contributed equally to this work.
}

check for

updates

Citation: Du, X.-L.; Xu, W.-J.; Shi, J.-L.; Guo, K.; Guo, C.-T.; Zheng, R.; Jiang, S.-W.; Chai, J. Glucocorticoid Receptor Alpha Targets SLC2A4 to Regulate Protein Synthesis and Breakdown in Porcine Skeletal Muscle Cells. Biomolecules 2021, 11, 721. https://doi.org/10.3390/ biom 11050721

Academic Editor: Menno Hoekstra

Received: 12 January 2021

Accepted: 28 April 2021

Published: 12 May 2021

Publisher's Note: MDPI stays neutral with regard to jurisdictional claims in published maps and institutional affiliations.

Copyright: (c) 2021 by the authors. Licensee MDPI, Basel, Switzerland. This article is an open access article distributed under the terms and conditions of the Creative Commons Attribution (CC BY) license (https:/ / creativecommons.org/licenses/by/ $4.0 /)$.

\begin{abstract}
In the presence of stress, the hypothalamic-pituitary-adrenal (HPA) axis activity can be enhanced to promote the secretion of a large amount of glucocorticoids (GCs), which play an important role in the anabolism and catabolism of skeletal muscle. When the endogenous and exogenous glucocorticoids are deficient or excessive, the body will produce stress-related resistance and change the protein metabolism. In this study, we investigated the effect of GC receptor GR $\alpha$ on protein breakdown and synthesis in porcine skeletal muscle cells (PSCs). Overexpression of GR $\alpha$ was shown to increase the expression of protein degradation-related genes, while knockdown of GR $\alpha$ decreased the expression of these genes. Additionally, we found a relationship between GR $\alpha$ and solute carrier family 2 member 4 (SLC2A4), SLC2A4 expression level increases when stress occurs, suggesting that increasing SLC2A4 expression can partially alleviate stress-induced damage, and we found that there is a combination between them via luciferase reporter assays, which still needs to be confirmed in further studies.
\end{abstract}

Keywords: pig; glucocorticoid; GR $\alpha$; protein deposition; SLC2A4

\section{Introduction}

Glucocorticoids (GCs) are a type of steroid hormones secreted by the adrenal cortical bundle, and their secretion could be increased under stress [1]. The feedback regulation of GCs on the hyperactivity of the hypothalamic-pituitary-adrenal (HPA) axis is considered to be mediated through two different intracellular receptors: the mineralocorticoid receptor (MR) and GC receptor (GR) [2]. GR, a family member of conserved nuclear receptors, is a nuclear transcription factor widely present in various somatic cells [3] and can only be activated under high concentration GC stimulation [4]. GR $\alpha$ and GR $\beta$ are two subtypes of GC. In most cells, GR $\alpha$ is more abundant than GR $\beta$, and it can bind to GC and activate the response of related genes $[5,6]$.

Under normal circumstances, insulin in the liver triggers the rapid uptake and oxidative decomposition of glucose [7], while under stress, GC acts as an antagonizing substance in the anabolic function of insulin to promote glycogen decomposition and gluconeogenesis in the liver [8-10]. Increasing the GC content can regulate the metabolism of glucose, fat and protein in the body [11]. Additionally, GC plays an important role in different substance metabolism. In skeletal muscle, GC reduces the protein synthesis rate and increases the protein breakdown rate, both of which can cause skeletal muscle atrophy [12,13]. Meanwhile, animal protein synthesis can be affected under chronic stress. In our previous study, the weight gain rate of cortisol-fed piglets was found to be significantly lower than that of the control group [14]. Stress hormones lead to slower growth in pigs, which is closely related to the rate of protein deposition and breakdown in muscles. Moreover, 
glucose is an important fuel for contracting muscle, and normal glucose metabolism is vital for muscle health. Glucocorticoids regulate multiple aspects of glucose homeostasis in skeletal muscle. Solute carrier family 2 member 4 (SLC2A4) plays an important part in glucose metabolism, which controls glucose transport into muscle tissues in response to insulin after exercise $[15,16]$. In our previous experiment on cortisol-fed piglets, we found that the expression of SLC2A4 in the cortisol group was significantly higher than that in the control group via transcriptome sequencing. Therefore, we speculate that stress may affect the decomposition or deposition of protein through GR $\alpha$ regulation of SLC2A4, leading to a slower growth rate.

In this study, we investigated the impact of GR $\alpha$ on protein deposition-related genes in porcine skeletal muscle cells (PSCs) and explored the relationship between GR $\alpha$ and SLC2A4.

\section{Results}

2.1. Effects of Glucocorticoid and Its Antagonist on Protein Deposition in Pig Skeletal Muscle Cells

Porcine skeletal muscle cells (PSCs) were cultured in 6-well plates and treated with four different Dexamethasone (DEX)concentrations $(0,0.1,1$ and $10 \mu \mathrm{M})$ for $48 \mathrm{~h}$. The highest expression of GR $\alpha$ was found to occur at $0.1 \mu \mathrm{M}$ DEX (Supplementary Materials Figure S1). Atrogin-1, FOXO1 and MSTN have been reported to affect protein deposition [17]. For further investigation, we used DEX and GC nuclear receptor antagonists (RU486) to treat PSCs. Based on the results of RT-PCR and Western blot, the mRNA expression of these genes increased when treated with DEX, but decreased after adding an equal concentration of RU486. We used the SUnSET non-radioactive method to detect the protein synthesis of the cells after treatment with DEX and its antagonist, and the protein synthesis rate was shown to decrease after adding DEX (Figure 1).

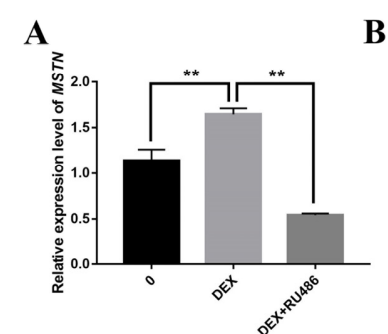

D



F

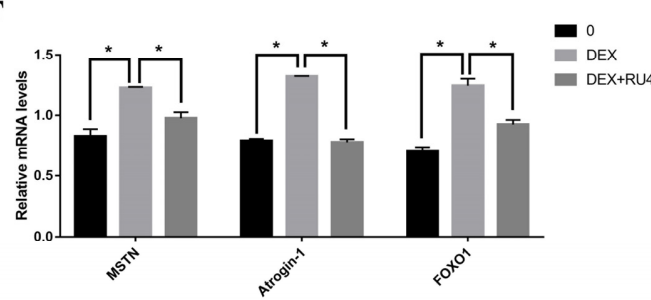

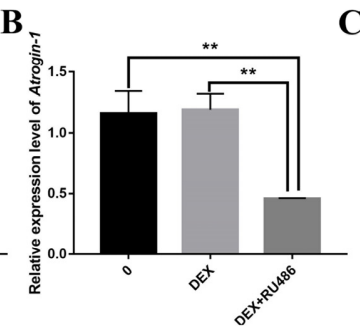

E
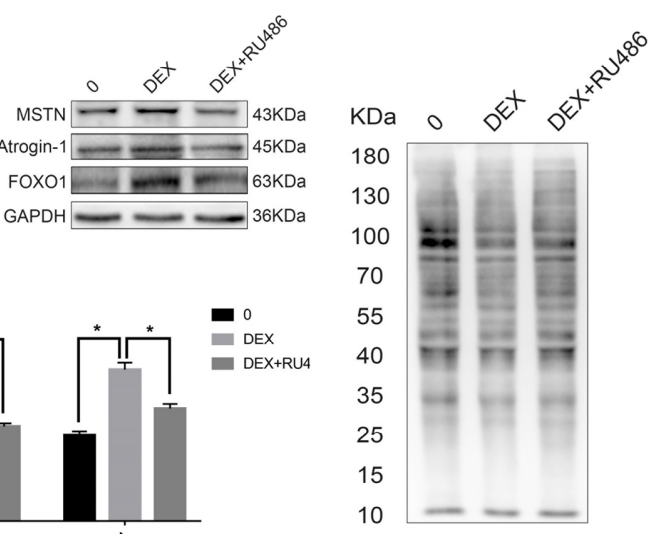

Figure 1. The influence of glucocorticoid and its antagonists on the expression of protein depositionrelated genes. (A-D) The mRNA expression levels of MSTN, Atrogin-1, FOXO1, IGF1 treated with DEX and RU486; (E) The protein expression levels of MSTN, Atrogin-1 and FOXO1 treated with DEX and RU486; (F) Quantification of Western blotting under the treatment of DEX and RU486; (G) Effect of glucocorticoid and its antagonist on the protein synthesis rate. ${ }^{*}, * *$ indicate significant difference at $p<0.05$ and $p<0.01$, respectively. 


\subsection{Effect of Glucocorticoid Receptor GRa on Protein Deposition}

Previous studies have shown that DEX affects the expression of protein depositionrelated genes in PSCs, so we further studied the effects of GR $\alpha$ on protein depositionrelated genes in PSCs by the overexpression and interference of GR $\alpha$. The results of overexpression and interference of GR $\alpha$ are shown in Figure 2. After overexpression of GR $\alpha$, the protein concentration was shown to decrease significantly in PSCs (Figure 3A), coupled with an increase in the expression of the inhibitory protein deposition-related gene and a decrease in the protein synthesis rate (Figure 4). After the intervention of GR $\alpha$, the protein concentration increased (Figure $3 \mathrm{~B}$ ), coupled with a decrease in the expression level of the inhibitory protein deposition-related genes and an increase in the protein synthesis rate (Figure 5). These results indicate that when stress occurs, both the level of glucocorticoids and the corresponding expression levels of their receptors are elevated, resulting in a decrease of protein deposition in the muscle.

A

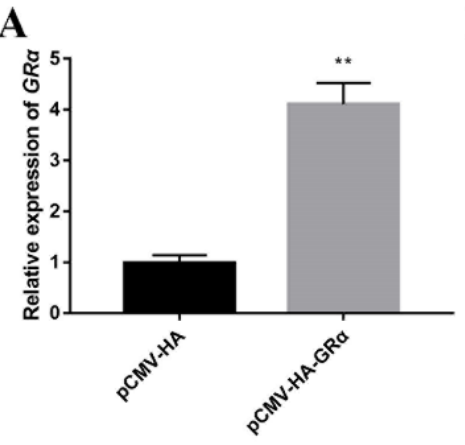

C

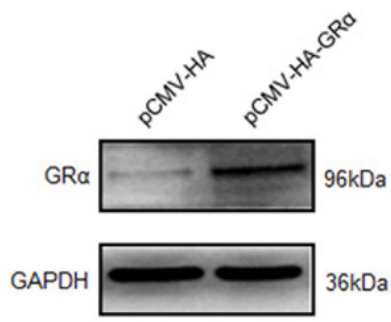

$\mathbf{E}$

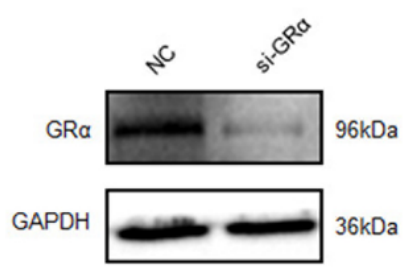

B

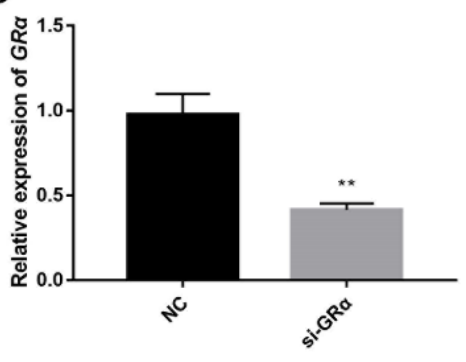

D

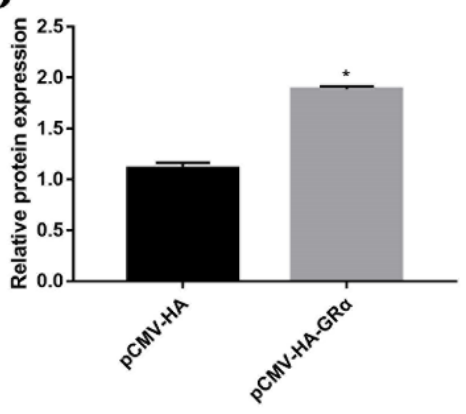

$\mathbf{F}$

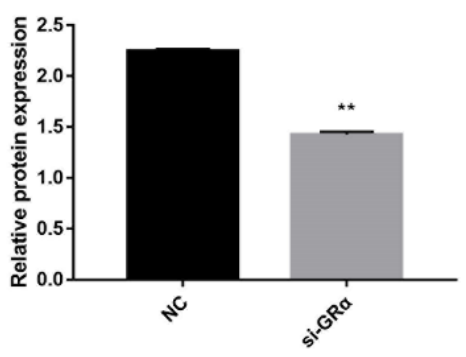

Figure 2. The expression level of GR $\alpha$ after its overexpression or interference in porcine skeletal muscle cells (PSCs). (A) The mRNA expression level of GR $\alpha$ after overexpression of GR $\alpha$; (B) The mRNA expression level of GR $\alpha$ after interference of GR $\alpha$; (C) The protein expression level of GR $\alpha$ after overexpression of GR $\alpha$; (D) Quantification of Western blotting after overexpression of GR $\alpha$; (E) The protein expression level of GR $\alpha$ after interference of GR $\alpha$; (F) Quantification of Western blotting after interference of GR $\alpha .{ }^{*}, * *$ indicate significant difference at $p<0.05$ and $p<0.01$, respectively. 
A

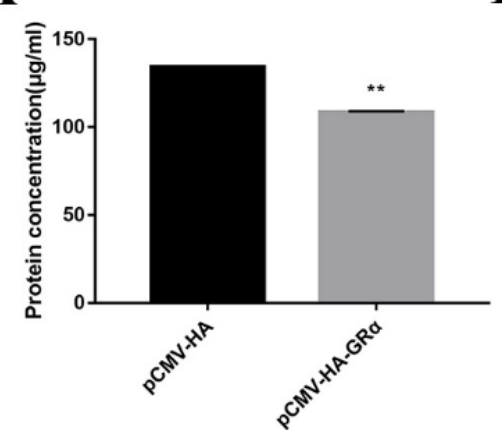

B

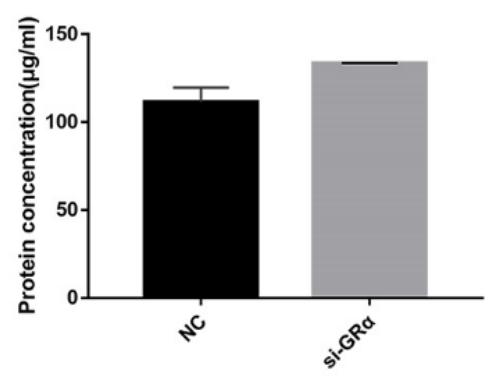

Figure 3. Changes in cellular protein concentration after overexpression or interference of GR $\alpha$. (A) Changes in cell protein concentration after overexpression of GR $\alpha$; (B) Changes in cellular protein concentration after interference of GR $\alpha .{ }^{* *}$ indicate significant difference at $p<0.01$.

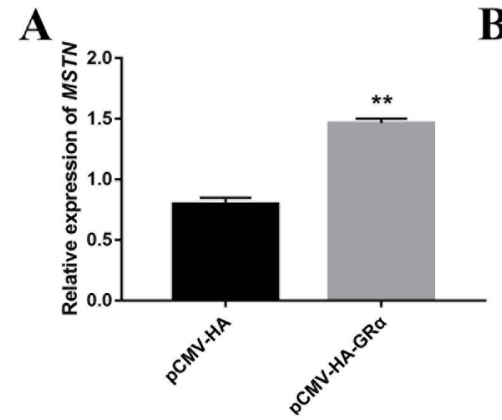

D

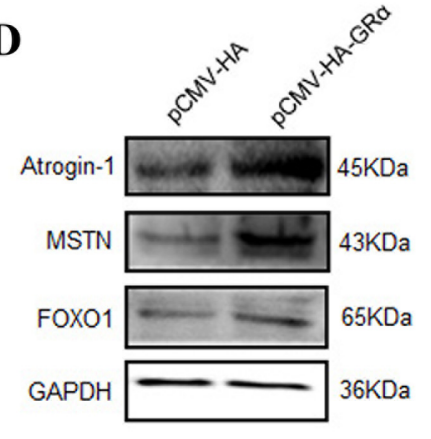

$\mathbf{F}$



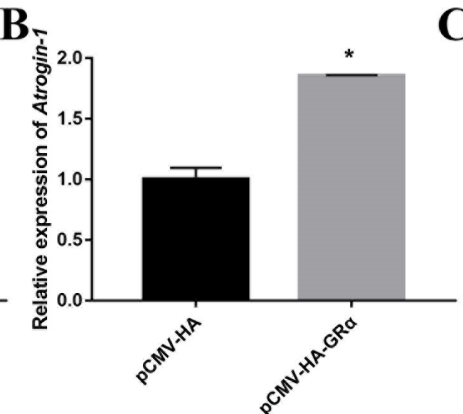

$\mathbf{E}$
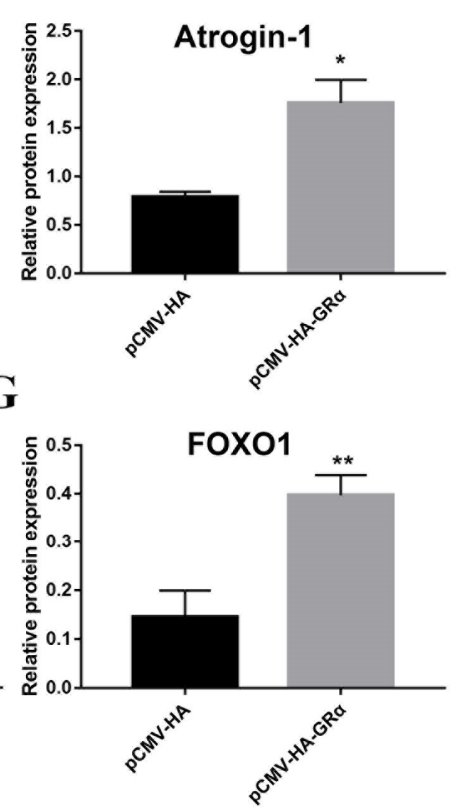

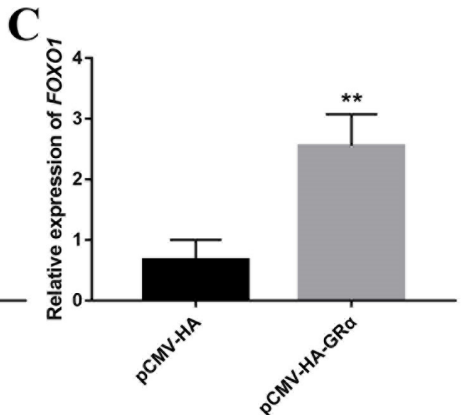

H

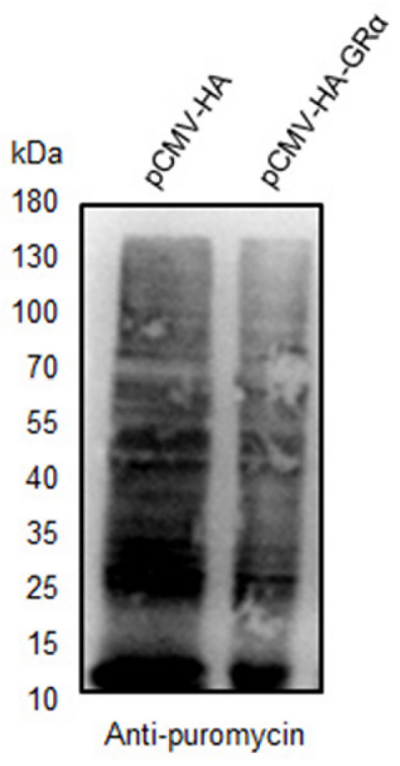

Figure 4. Changes of mRNA and protein levels of protein deposition-related genes. (A-C) The mRNA expression levels of protein deposition-related genes MSTN, Atrogin-1 and FOXO1 after overexpression of GR $\alpha$; (D) The protein expression level of protein deposition-related genes MSTN, Atrogin-1 and FOXO1 after overexpression of GR $\alpha$; (E-G) Quantification of Western blotting after overexpression of GR $\alpha$; $\mathbf{H})$ Effect of GR $\alpha$ overexpression on protein synthesis rate. *, ${ }^{* *}$ indicate significant difference at $p<0.05$ and $p<0.01$, respectively. 

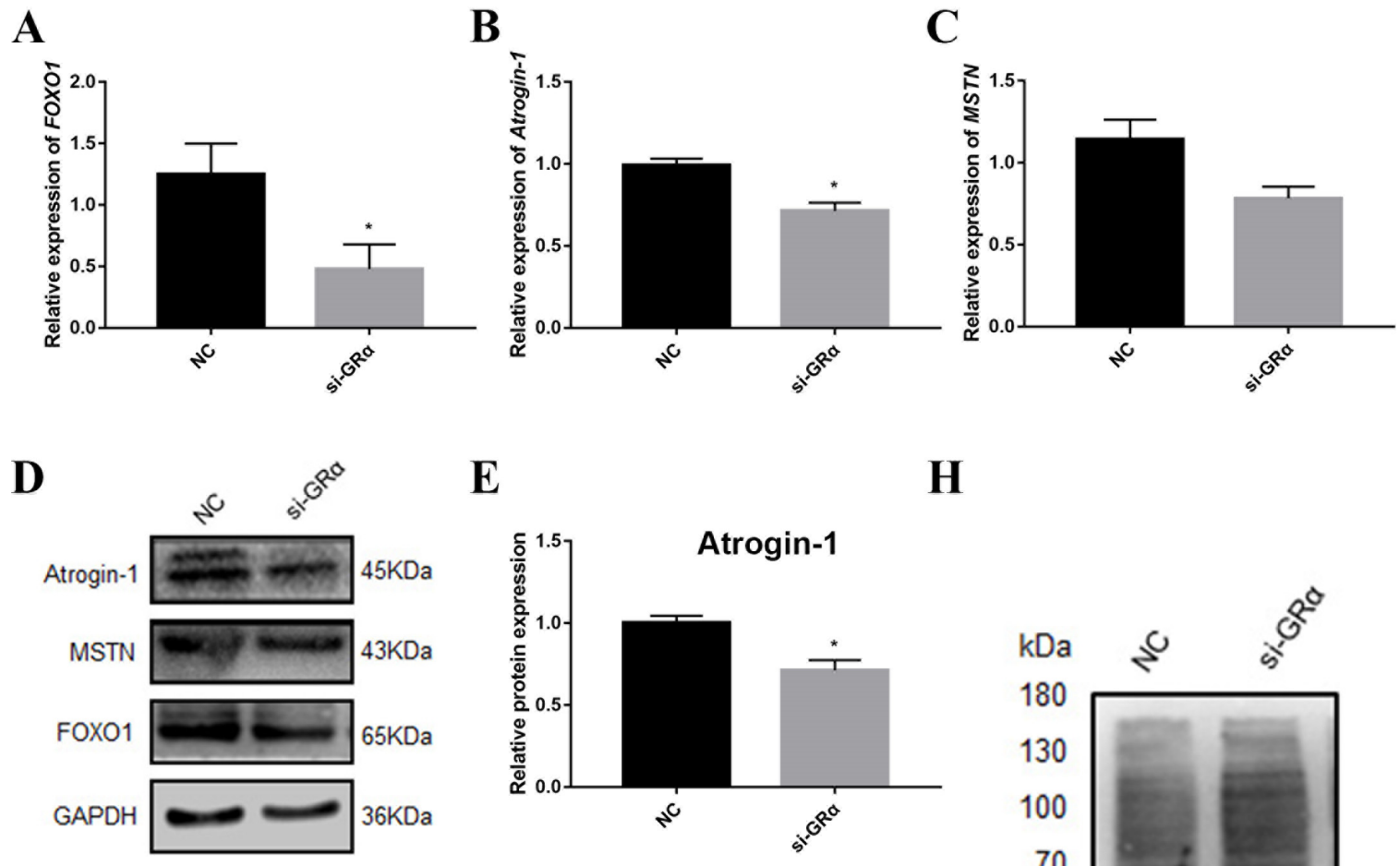

$\mathbf{E}$

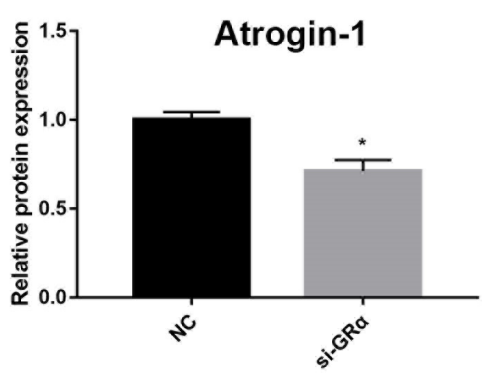

F

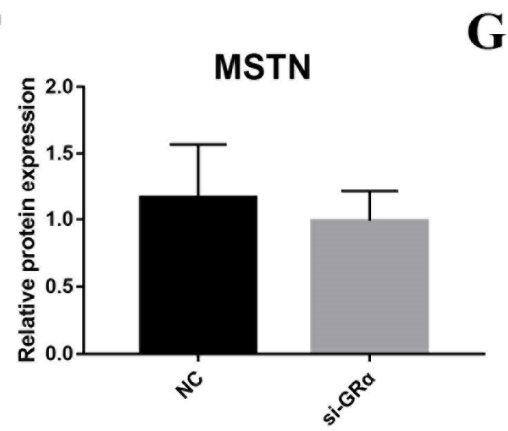

G

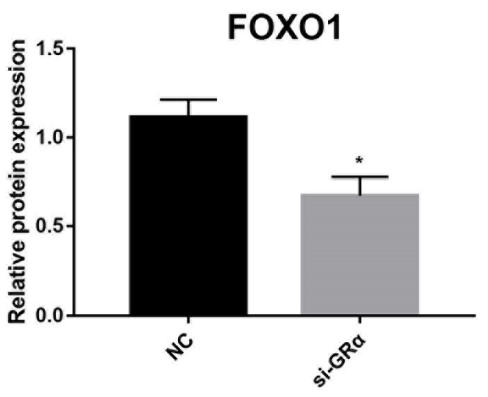

H

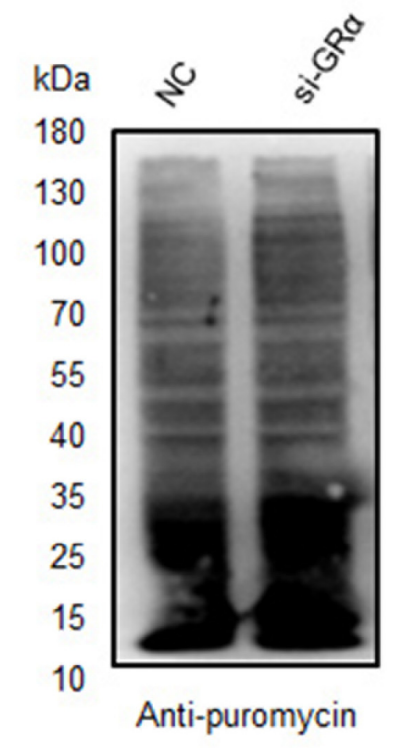

Figure 5. Changes of mRNA and protein levels of protein deposition-related genes after interference of GR $\alpha$. (A-C) The mRNA expression level of protein deposition-related genes FOXO1, Atrogin-1 and MSTN after interference of GR $\alpha$; (D) The protein expression levels of protein deposition-related genes MSTN, Atrogin-1 and FOXO1 after interference of GR $\alpha$; (E-G) Quantification of Western blotting after interference of GR $\alpha$. (H) Effect of interference of GR $\alpha$ on protein synthesis rate. ${ }^{*}$ indicate significant difference at $p<0.05$.

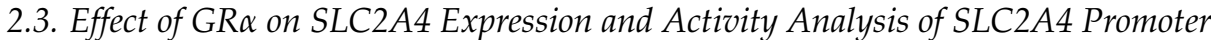

The differentially expressed genes were screened according to the sequencing results of the previous transcriptome analysis. The mRNA expression levels of SLC2A4, FKBP5 and GPX2 were markedly increased, in contrast to no significant difference in the expression level of FHL3 and USP18 in the longissimus dorsi muscle of cortisol-fed piglets versus the control group (Supplementary Materials Figure S1). The potential effect of GR $\alpha$ on differentially expressed genes was explored by overexpression and interference of GR $\alpha$ in porcine skeletal muscle cells. Overexpression of GR $\alpha$ significantly increased the mRNA and protein level of the SLC2A4 gene, while interference of GR $\alpha$ significantly decreased its mRNA and protein level (Figure 6). This indicates a certain relationship between GR $\alpha$ and $S L C 2 A 4$, i.e., the increase in the level of glucocorticoids in the body can promote the expression of SLC2A4, thereby regulating the response in the body. 
A

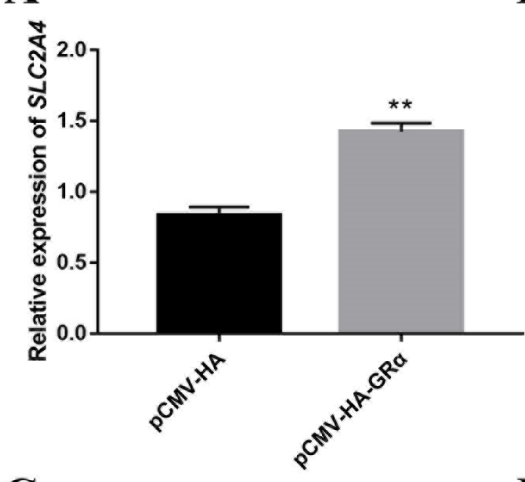

C

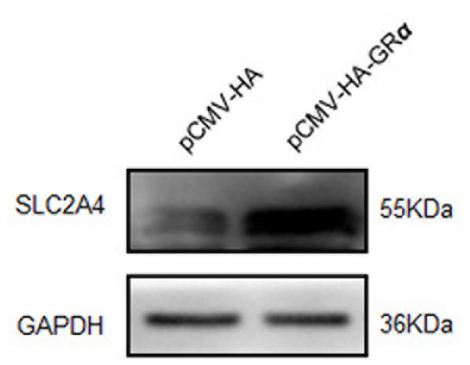

E

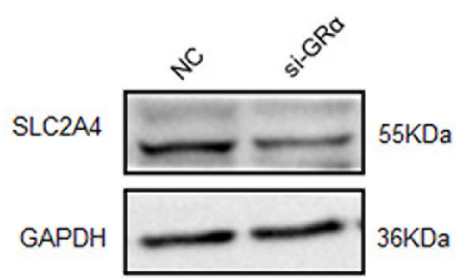

B

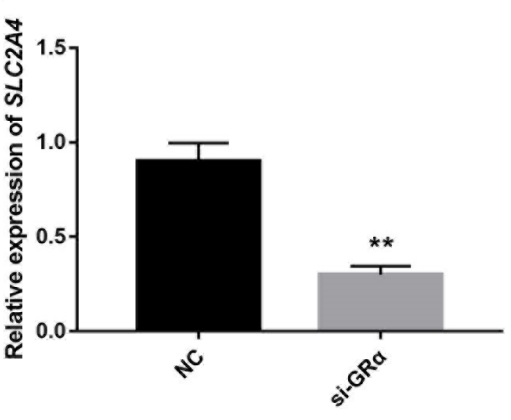

D
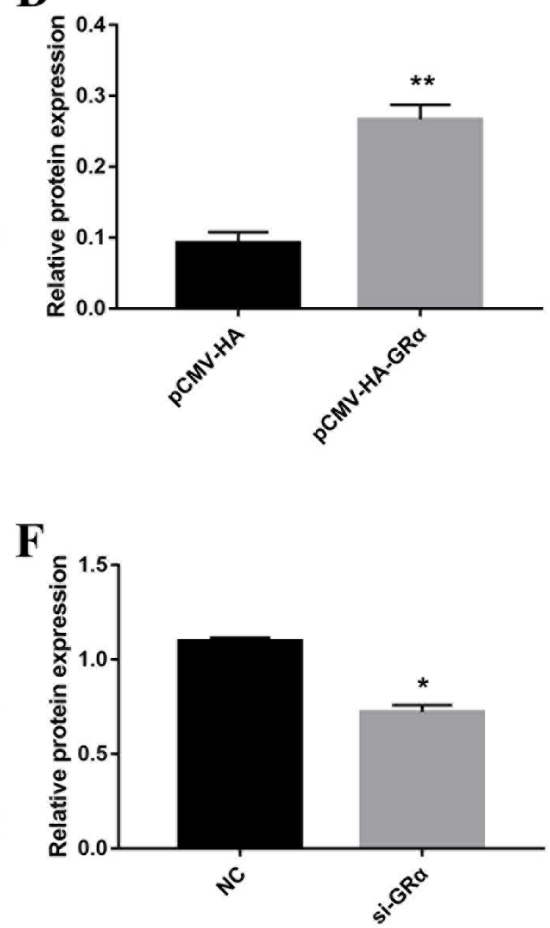

Figure 6. Changes in SLC2A4 protein level after overexpression or interference of GR $\alpha$. (A) The mRNA expression level of $S L C 2 A 4$ after overexpression of GR $\alpha$; (B) The mRNA expression level of $S L C 2 A 4$ after interference of GR $\alpha$; C: The protein expression level of SLC2A4 after overexpression of GR $\alpha$; (D) Quantification of Western blotting after overexpression of GR $\alpha$; (E) The protein expression level of SLC2A4 after interference of GR $\alpha$; (F) Quantification of Western blotting after interference of GR $\alpha .{ }^{*}{ }^{* *}$ indicate significant difference at $p<0.05$ and $p<0.01$, respectively.

The active site in the SLC2A4 promoter was predicted using the Neural Network Promoter Prediction (NNPP) online software, and the binding site of the transcription factor GR $\alpha$ and SLC2A4 was predicted with the 1167 bp sequence containing the promoter active site using the JASPAR online software (Figure S2). Four deletion fragments upstream of the translation initiation site were amplified based on the predicted binding site, which were $1167 \mathrm{bp}, 827 \mathrm{bp}, 487 \mathrm{bp}$, and $294 \mathrm{bp}$, and each was inserted into the pGL3-Basic carrier, named S1, S2, S3 and S4, respectively. The activity of the deleted fragments was determined by transfecting them into pig embryonic kidney cells (PK) and PSC. The luciferase reporter system was used to detect the dual-luciferase activity of these fragments. The S3 fragment was detected to be most active (Figure 7A,B), so the S3 fragment and the GR $\alpha$ overexpression vector were co-transfected for further analysis. Compared with the control group, the co-transfection group showed a significant increase in activity (Figure 7C,D), indicating that the S3 fragment has a site binding to GR $\alpha$. To determine 
whether GR $\alpha$ directly binds to SLC2A4, we verified a GR binding site on the S3 fragment through site-directed mutagenesis (Figure 7E). Meanwhile, we found that the mutation of the binding site did not completely reduce the activity of the luciferase reporter, indicating that there might be other GR $\alpha$ binding sites or other modes of action on the S3 fragment.

A

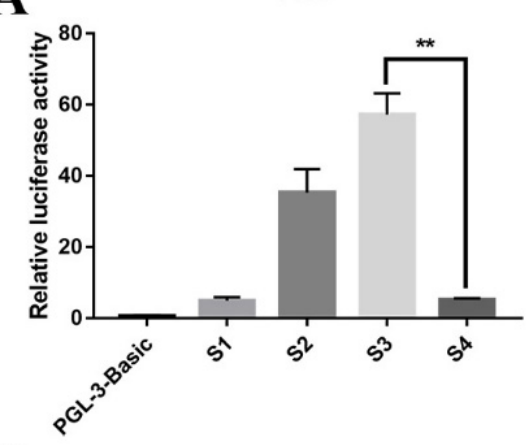

C

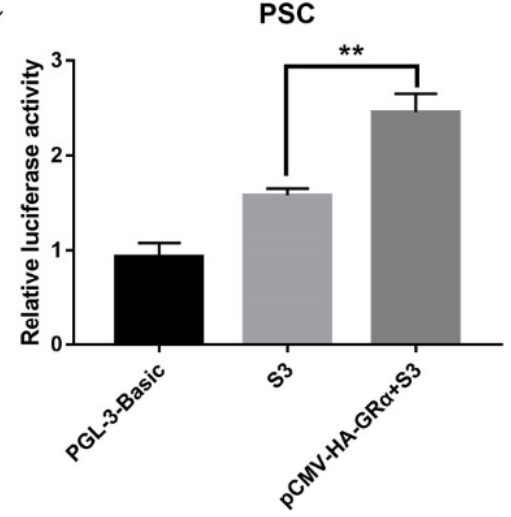

E

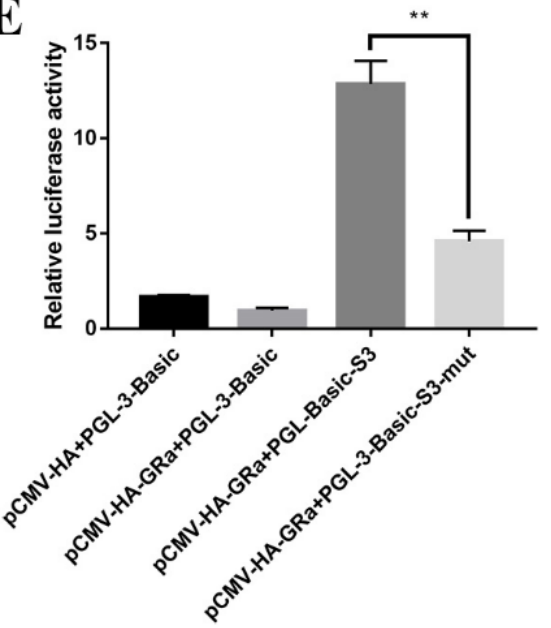

B



D

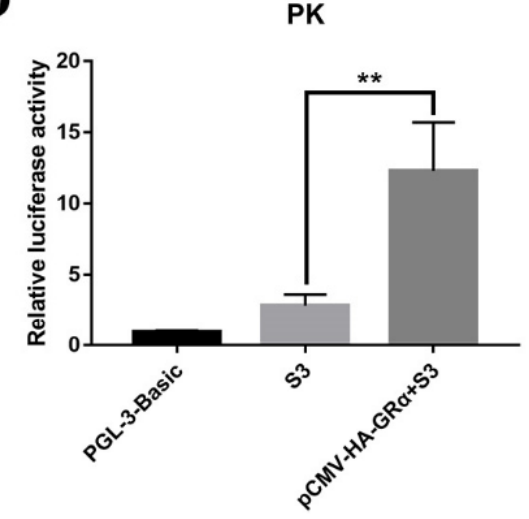

Figure 7. Activity analysis of different deletion fragments of $S L C 2 A 4$. (A) The relative luciferase activity of the SLC2A4 deletion fragment vector in porcine skeletal muscle cells; (B) The relative luciferase activity of the deletion fragment of SLC2A4 promoter in pig embryonic kidney cells (PK); (C) The relative luciferase activity after co-transfection of S3 recombinant vector and pCMV-HA-GR $\alpha$ into PSC; (D) The relative luciferase activity after co-transfection of S3 recombinant vector and pCMV-HA-GR $\alpha$ into PK. (E) Luciferase assays were conducted into PK cells co-transfected with GR $\alpha$ and PGL-3-Basic reporter containing either wild-type (500 ng) or mutant SLC2A4 binding site (Mut) $(500 \mathrm{ng}) .{ }^{* *}$ indicate significant difference at $p<0.01$. 


\subsection{Effect of SLC2A4 on Protein Deposition}

In order to study the role of SLC2A4 in protein deposition, an overexpression vector of SLC2A4 was constructed, and the expression levels of Atrogin-1, MSTN, FOXO1 and IGF-1 were detected after overexpression of SLC2A4 in PSC. The expression level of Atrogin1 was shown to decrease significantly, in contrast to an increase in the expression of FOXO1 and MSTN (Figure 8A-E). The protein expression level of Atrogin-1 was detected to decrease significantly (Figure $8 \mathrm{~F}-\mathrm{H}$ ). Under low glucose and hypoxia conditions, the AMPK signaling pathway was activated, thereby inhibiting the mTOR signaling pathway. Overexpression of SLC2A4 and analysis of the genes in the mTOR signaling pathway revealed an increase in the phosphorylation level of mTOR and its downstream genes S6K1 and 4EBP1, indicating that the increased expression of the glucose transporter SLC2A4 can alleviate the decrease of protein deposition caused by stress (Figure 8I).
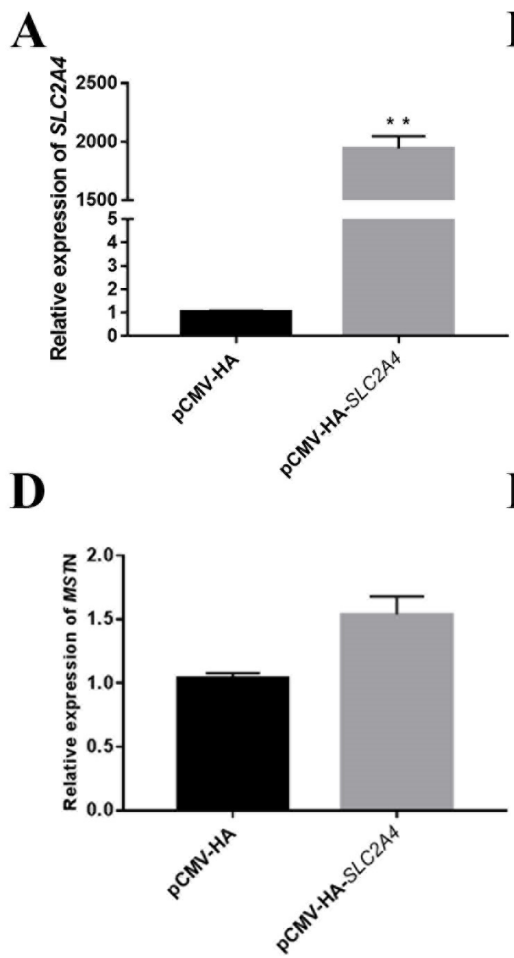

G



B

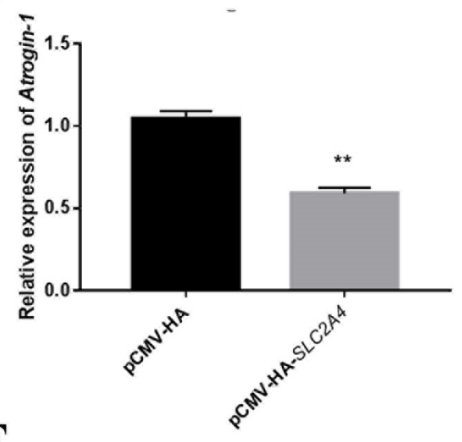

E

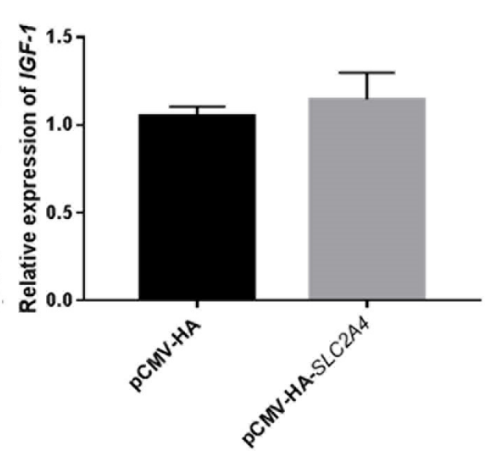

H

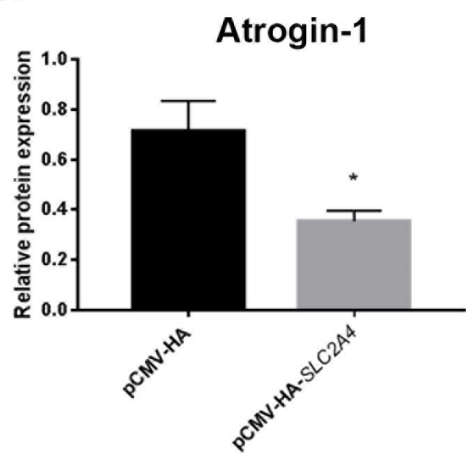

C
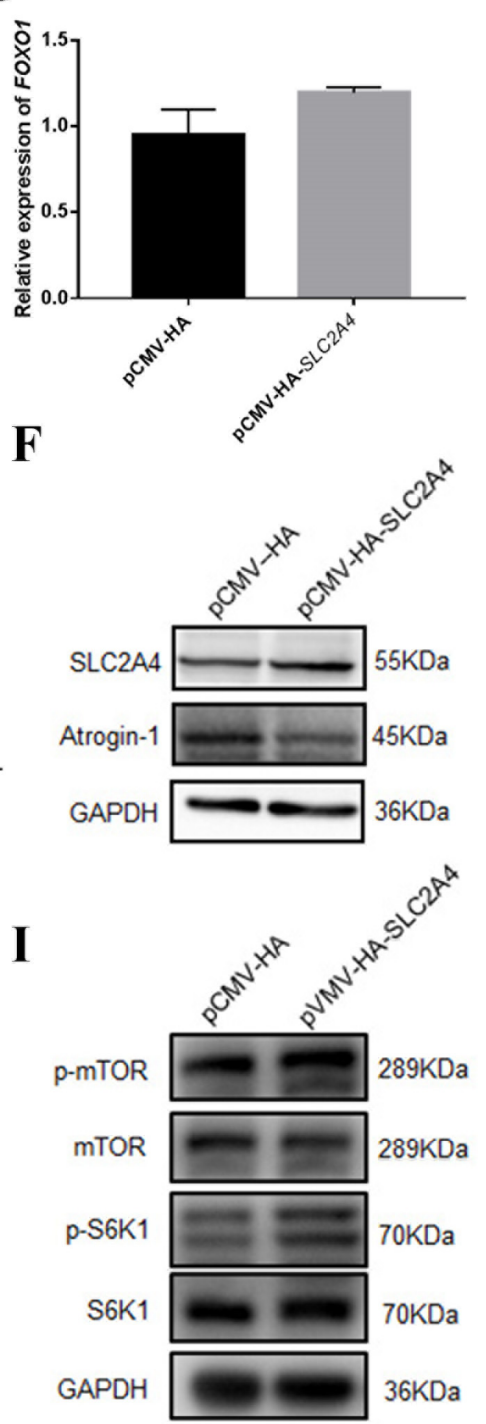

Figure 8. The expression of genes involved in protein deposition and mTOR signaling pathway after overexpression of SLC2A4. (A-E) The mRNA expression levels of SLC2A4, MSTN, Atrogin-1, FOXO1 and IGF1 after overexpression of SLC2A4; (F) The protein expression level of $S L C 2 A 4$ and Atrogin-1 after overexpression of GR $\alpha ;(\mathbf{G}, \mathbf{H})$ Quantification of Western blotting after overexpression of SLC2A4; (I) The phosphorylation level of mTOR and S6K1 in mTOR signaling pathway after overexpression of $S L C 2 A 4 .{ }^{*},{ }^{* *}$ indicate significant difference at $p<0.05$ and $p<0.01$, respectively. 


\section{Discussion}

There are a variety of stresses in the growth and development of livestock. The occurrence of stress will induce a series of reactions in the body, such as the increase in the level of free radicals in the cells, and the body's antioxidant system such as SOD cannot completely remove these free radicals, causing cell apoptosis, tissue damage, increased muscle fiber gap, and decreased meat quality $[14,18]$. In addition, when stress occurs, the body's hormone secretion is abnormal, thus reducing the digestion and absorption capacity of nutrients, which in turn affects the nutrient utilization rate and growth performance of the body [19]. The neuroendocrine system plays an important role in maintaining homeostasis, and glucocorticoids and insulin perform important functions in maintaining energy metabolism in the body [20]. Under external environmental stresses, such as preslaughter stress, transport stress, etc., the HPA axis can increase the level of glucocorticoids in the blood through interaction with insulin, adrenaline, glucagon and sympathetic nerves, leading to decreased insulin levels, mobilization of fat and muscle tissue, and enhanced hepatic gluconeogenesis [21]. Glucocorticoids can reduce the level of protein synthesis, and many genes are reported to affect protein synthesis, such as Atrogin-1, MSTN, FOXO1 and IGF-1 [17]. Among them, the Atrogin-1 gene has a high level of expression in the event of muscle atrophy. MSTN is an important factor regulating muscle growth and development, and an increase in its expression will cause small muscle mass blocks in animals $[22,23]$. The FOXO family is involved in the regulation of various cellular functions, such as transcriptional metabolism, apoptosis and cell cycle. In this family, FOXO1 can regulate cell differentiation. When constructing a specific transgenic mouse overexpressing FOXO1, the mutant mouse was found to be lower than the wild-type mouse in body weight and skeletal muscle weight, and higher in protein degradation in skeletal muscle [24-26]. In this study, we investigated the effect of glucocorticoids on protein deposition by simulation on PSC, and found that the related genes varied in their expression. Specifically, when the stress state leads to an increase in the glucocorticoid level, the protein synthesis in the body will decrease, and so will protein deposition.

Despite the potential involvement of calcium-dependent protein degradation, the glucocorticoid-stimulated muscle protein breakdown is primarily caused by ubiquitinproteasome-dependent proteolysis, and under certain catabolic conditions, such as sepsis, the interaction between glucocorticoids and pro-inflammatory cytokines is also important for stimulating muscle protein breakdown. In a previous study on muscle atrophy, the activation of muscle protein degradation was found to require endogenous glucocorticoids. Specifically, when given the physiological doses of glucocorticoids, the adrenalectomized mice showed decreased PI3K activity and progressive muscle atrophy. These reactions are related to an increase in GR $\alpha$ expression. The muscle-specific deletion of GR $\alpha$ in mice showed an increase in the activity of PI3K, and the non-genomic effects of GR are assumed to contribute to the activation of muscle protein degradation [27]. In this study, overexpression of GR $\alpha$ reduced the protein concentration, suggesting the decrease of protein deposition after overexpression of GR $\alpha$. Analysis of the mRNA and protein expression levels of genes related to protein degradation revealed an increase in their expression after overexpression of GR $\alpha$, while an increase in their protein concentration and a decrease in their expression after the interference of GR $\alpha$. This result supported our hypothesis that GR promotes muscle protein degradation and reduces protein deposition.

$S L C 2 A 4$ is an insulin-sensitive transporter that can be inserted into the intracellular vesicle membrane and translocated to the plasma membrane under insulin stimulation, thereby increasing the glucose uptake of cells. Muscle contraction can stimulate the translocation of SLC2A4 in skeletal muscle and induce a large translocation when contraction is tight $[28,29]$. Under chronic stress, high levels of glucocorticoids in the body can cause high blood pressure. Zheng Xiang et al. detected the blood glucose levels in mice after injection of DEX, and found an increase in the blood glucose levels of the experimental group [30,31]. Meanwhile, we also found that the expression level of SLC2A4 was increased in the longissimus dorsi muscle samples of cortisol-fed piglets or after overexpression of 
GR $\alpha$ in PSC. The gene transcription is promoted by the combination of glucocorticoid and glucocorticoid reaction element of the gene promoter region [32]. The expression of SLC2A4 was increased by overexpression of GR $\alpha$, but decreased by the interference of $\mathrm{GR} \alpha$, inferring that GR $\alpha$ may also have a relationship with $S L C 2 A 4$. For further analysis, we predicted a binding site for GR $\alpha$ on SLC2A4, which was investigated by dual-luciferase reporter and co-transfection with GR $\alpha$ overexpression vector. We verified that there is a GR $\alpha$ binding site on the S3 fragment through site-directed mutagenesis (Figure 7E), and found that the mutation of the binding site could not completely reduce the activity of the luciferase reporter, indicating that there may be other GR $\alpha$ binding sites or other modes of action on the $\mathrm{S} 3$ fragment. GR $\alpha$ controls transcription by two major modes of action: (1) binding GR $\alpha$ homodimers to glucocorticoid response elements (GREs) in the regulatory sequences of GR $\alpha$ target genes, and (2) modulating the activity of other transcription factors, such as AP-1, NF- $\mathrm{kB}$ and Stat5, independently of direct DNA contact, a process designated as cross-talk, which has been previously confirmed [33] (Reichardt et al., 2016). So we speculated that there might exist a second mode of action between GR $\alpha$ and SLC2A4, and it needs to be illustrated by our further experiment. It was initially shown that GR $\alpha$ binds to SLC2A4 and acts to increase SLC2A4 expression and glucose transport level to alleviate the effects of glucocorticoid-induced reduction in protein deposition. SLC2A4 is in the insulin signaling pathway, and skeletal muscle is the main site of insulin-stimulated glucose uptake. Glucose transport is found to increase in response to the contraction in isolated skeletal muscle, indicating that the signal transduction pathway caused by the deficiency of internal cellular energy partially acts on contraction [34]. In our study, overexpression of SLC2A4 was found to decrease the expression of the proteolytic gene Atrogin-1, while increasing the expression of the other two genes (FOXO1 and MSTN), indicating that glucose stress can partially alleviate the effects of protein deposition under stress. We think a feedback mechanism may exist: under stress, the body will reduce protein synthesis, but will perform feedback regulation to make the body as balanced as possible. Buffering provides an alternative regulatory strategy to negative feedback [35-37]. Additionally, the mTOR signaling pathway plays an important role in signaling pathways related to protein synthesis. mTOR promotes protein synthesis through phosphorylation of two key effectors, p70S6 kinase (S6K1) and elF4 binding protein (4EBP) [38]. By overexpressing SLC2A4, we detected the mRNA expression in the mTOR signaling pathway and then found an increase in the phosphorylation level of mTOR and S6K1 protein. In a previous study on muscle atrophy, mTOR activation significantly decreases the expression level of Atrogin-1 and efficiently counteracts the catabolic processes provoked by glucocorticoids [39], implying the SLC2A4 reduces the expression level of Atrogin-1 through the mTORC1 signaling pathway, thus alleviates the decrease in protein deposition caused by stress.

In summary, the HPA axis of the body is shown to be activated during stress, leading to increased levels of glucocorticoids and their receptors, which increases the expression of genes Atrogin-1, MSTN and FOXO1 and promotes protein breakdown, resulting in reduced protein synthesis and inhibition of protein deposition. Meanwhile, elevated levels of glucocorticoids can increase the expression of the glucose transporter gene SLC2A4. Overexpressed SLC2A4 promotes mTOR signal and reduces the expression level of the protein degradation gene Atrogin-1, which partially alleviates the decrease of protein deposition in porcine skeletal muscle cells (Figure 9). 




Figure 9. The schematic diagram of glucocorticoid regulating protein deposition process.

4. Materials and Methods

4.1. Animals and Samples

The big white piglets of 2-3 days were provided by the Farm of Huazhong Agricultural University (Wuhan, China). After the piglets were slaughtered, the longissimus dorsi and leg muscles were taken to separate for the primary skeletal muscle cells. Finally, the skeletal muscle cells were grown in incubators at $37{ }^{\circ} \mathrm{C}$ and $5 \% \mathrm{CO} 2$, and proliferating cells were cultured in Dulbecco's Modified Eagle's Medium (DMEM) supplemented with 10\% fetal bovine serum (DMEM, Gibco, Grand Island, NY, USA).

Animals: All experimental animal procedures in this study were performed according to the guidelines of Good Laboratory Practice, and the animals were supplied with nutritional food and sufficient water. Animal feeding and tests were conducted based on the National Research Council Guide for the Care and Use of Laboratory Animals and approved by the Institutional Animal Care and Use Committee at Huazhong Agricultural University.

Isolation and culture of primary skeletal muscle cells: Primary myoblasts were isolated and cultured as described previously [40]. Primary skeletal muscle cells were isolated from the longissimus dorsi skeletal muscles of 2-3 day old piglets, minced and digested in a mixture of type I collagenase and DMEM (DMEM, Gibco, Grand Island, NY, USA).

\subsection{Total RNA Preparation and cDNA Synthesis}

Total RNA was isolated at $48 \mathrm{~h}$ after cell transfection, using total RNA extraction kit (Omega bio-tek, Norcross, GA, USA) according to the manufacturer's protocol. The RNA integrity was checked using denaturing gel electrophoresis and the RNA concentration was measured with a NanoDrop 2000 spectrophotometer (Thermo Scientific, Waltham, MA, USA). Total RNA was reverse transcribed using a RevertAid ${ }^{\mathrm{TM}}$ First Strand cDNA Synthesis Kit (Thermo Scientific, Waltham, MA, USA).

\subsection{Quantitative Real-Time PCR}

The specific fluorescent quantitative PCR primers were designed using cDNA as a template; the mRNA level was quantified using $\beta$-actin gene as an internal reference; the real-time quantitative PCR experiments were performed on the CFX384 Touch $^{\mathrm{TM}}$ fluorescence quantitative PCR instrument using SYBR Green qPCR Master Mix (Bio-Rad). Finally, the $2^{-\Delta \Delta \mathrm{Ct}}$ method was used for data analysis, and one-way analysis of variance was performed to determine the significance at $p<0.05$ (significant) and $p<0.01$ (extremely significant). Primer sets are listed in Table 1. 
Table 1. Primer sequences used for qPCR.

\begin{tabular}{|c|c|c|}
\hline Gene & Sequence of Primer $\left(5^{\prime}-3^{\prime}\right)$ & Amplicon Size (bp) \\
\hline $\mathrm{GR} \alpha$ & $\begin{array}{l}\text { F:GCTTGCGGTGGACTTTC } \\
\text { R:AGGTCACTTCCCATCACTTTA }\end{array}$ & 126 \\
\hline MSTN & $\begin{array}{l}\text { F:TACCCTCACACTCATCTTGTGC } \\
\text { R:ACCCACAGCGATCTACTACCA }\end{array}$ & 160 \\
\hline Atrogin-1 & $\begin{array}{l}\text { F:CAAAGGCTAAGTGATGGCCG } \\
\text { R:GAGGGTAGCATCGCACAAGT }\end{array}$ & 205 \\
\hline FOXO1 & $\begin{array}{l}\text { F:AATCGAGTTACGGAGGCATGG } \\
\text { R:TAGGGCCCATCAGCACATTC }\end{array}$ & 165 \\
\hline$S L C 2 A 4$ & $\begin{array}{l}\text { F:TCTCTGTGGGTGGCATGTTC } \\
\text { R:TAGGCACCAATGAGGAACCG }\end{array}$ & 181 \\
\hline FKBP5 & $\begin{array}{l}\text { F:GATGGAGTACGGCTTGTCAG } \\
\text { R:CAAGCCCTTCTCATTGGCAC }\end{array}$ & 166 \\
\hline FHL3 & $\begin{array}{l}\text { F:CTGTGCAAAATGCAGCGAGT } \\
\text { R:GGAAGTGGCGATCCTCGTAA }\end{array}$ & 167 \\
\hline USP18 & $\begin{array}{l}\text { F:TACCTCACCGTCTGGAACCT } \\
\text { R:CAGGGGCTTTGAGTCCATGT }\end{array}$ & 195 \\
\hline IGF1 & $\begin{array}{l}\mathrm{F}: \text { СТCTCСTTCACCAGCTCTGC } \\
\text { R:TCCAGCСTCCTCAGATCACA }\end{array}$ & 200 \\
\hline$\beta$-actin & $\begin{array}{l}\text { F:CCAGGTCATCACCATCGG } \\
\text { R:CCGTGTTGGCGTAGAGGT }\end{array}$ & 158 \\
\hline
\end{tabular}

\subsection{BCA Method for Detecting Protein Concentration}

The protein concentration was determined using the BCA Protein Concentration Test Kit (Thermo Fisher Scientific, Waltham, MA, USA) according to the procedure provided by the supplier.

\subsection{Plasmid Construction, siRNA Synthesis, and Cell Transfection}

Briefly, For the GR $\alpha$ and SLC2A4 overexpression plasmids, full-length sequences were cloned into the pCMV-HA plasmid. Meanwhile, various amounts of SLC2A4 promoter sequences were amplified from pig genomic DNA and cloned into the pGL3-Basic vector to generate luciferase reporter plasmids. Full-length GR $\alpha$ and SLC2A4 sequences were amplified with full-length-F/R primers (Table 2) and truncated SLC2A4 sequences were amplified with $S L C 2 A 4-F / R$ primers (Table 2). GR $\alpha$ siRNA was synthesized by Shanghai GenePharm Company, using the following GR $\alpha$ siRNA sequences: GR $\alpha$ siRNA (sense): GCUACUCAAGCCCUGGAAUTT; GR $\alpha$ siRNA (antisense): AUUCCAGGGCUUGAUCAGCTT. For cell transfection, primary cells were transfected with $4 \mu \mathrm{g}$ of expression vectors or $10 \mu \mathrm{L}$ of siRNA oligo using Lipofectamine 2000 (Invitrogen, Carlsbad, CA, USA) in each well of a 6-well plate.

\subsection{Western Blot Analysis}

Briefly, PSC was washed with PBS and lysed in RIPA lysis buffer (Beyotime Biotechnology Company, Shanghai, Chian). Next, $20 \mu \mathrm{g}$ of total protein was resolved by $10 \%$ sodium dodecyl sulfate-polyacrylamide gel electrophoresis and electro-transferred onto a poly-vinylidene fluoride (PVDF) (Millipore, Burlington, MA, USA) membrane. The PVDF membrane was blocked in 5\% skim milk powder dissolved in TBST for 90 min at room temperature. Primary antibodies were applied in sealing fluid at $4{ }^{\circ} \mathrm{C}$ overnight. Subsequently, the PVDF membrane was washed with TBST and stained with the appropriate HPR-labeled secondary antibodies (goat anti-rabbit or mouse) for $1 \mathrm{~h}$ at room temperature. After washing with TBST, the membrane was analyzed using the ECL Reagent (Beyotime Biotechnology). The antibodies used included: GAPDH (GB11002) antibody pur- 
chased from Servicebio, Wuhan, China; $\beta$-Actin (AC037), MSTN (A6913), SLC2A4 (A7637), Atrogin-1 (MAFbx) (A3193), GR $\alpha$ (NR3C1) (A2164) and secondary antibody purchased from Abclonal Technology, Wuhan, Chian; P-S6K1 (AF3228, Affinity Biosciences), P-mTOR (S2448, Cell Signaling Technology, Danvers, MA, USA), and mouse puromycin antibody 12D10 (320591, Millipore).

Table 2. Primers used for plasmid construction.

\begin{tabular}{|c|c|}
\hline Gene & Sequence of Primer( $\left(5^{\prime}-3^{\prime}\right)$ \\
\hline \multirow{2}{*}{ GR $\alpha$-full length sequence } & F: ATGGACCCCAAGGAATCGCTGACCC \\
\hline & R: TCACTTTTGATGAAACAGAAGTTTT \\
\hline \multirow{2}{*}{ SLC2A4- full length sequence } & F: GGCTACACCTGTGGCATATG \\
\hline & R: CTTGTCTTAGGAGCTGGAGG \\
\hline \multirow{2}{*}{ SLC2A4-S1-1167bp fragment } & F: CGGGGTACCGAGGCCCGTTTTCCCAGCCG \\
\hline & R: CCGCTCGAGCTTGTCTTAGGAGCTGGAGG \\
\hline \multirow{2}{*}{ SLC2A4-S2-827bp fragment } & F: CGGGGTACCCTAGGAACGGAATTTCCTGT \\
\hline & R: CCGCTCGAGCTTGTCTTAGGAGCTGGAGG \\
\hline \multirow{2}{*}{ SLC2A4-S3-487bp fragment } & F: CGGGGTACCGTGGGCGGAGTCTTCGCACT \\
\hline & R: CCGCTCGAGCTTGTCTTAGGAGCTGGAGG \\
\hline \multirow{2}{*}{ SLC2A4-S4-294bp fragment } & F: CGGGGTACCCTTCTGGGGTGTGCGGGCT \\
\hline & R: CCGCTCGAGCTTGTCTTAGGAGCTGGAGG \\
\hline \multirow{2}{*}{ SLC2A4-S3-mut fragment } & F: TCGCCCCTACTGACTTCTGCCCGCCAGGCT \\
\hline & R: GGGCAGAAGTCAGTAGGGGCGACGGGGG \\
\hline
\end{tabular}

\subsection{SUnSET Non-Radioactive Method for Detecting Protein Synthesis Rate}

In this study, the surface sensing of translation (SUnSET) non-radioactive method was used to measure the protein synthesis rate of porcine skeletal muscle cells [40]. After transfection with GR $\alpha$ overexpression vector and GR $\alpha$ interference fragment, the cells were treated separately with DEX and RU486 for $48 \mathrm{~h}$, followed by the addition of $1 \mu \mathrm{g} / \mathrm{mL}$ puromycin (Beyotime Biotechnology, Shanghai, China) and treatment of $30 \mathrm{~min}$. Finally, the change of puromycin was detected using Western blot.

\subsection{Luciferase Reporter Assays}

PSC was seeded in 24-well plates until reaching sub-confluence, followed by transient co-transfection with a luciferase reporter plasmid (500 ng/well) containing the conserved binding sequence of GR $\alpha$ for detecting the GR $\alpha$ activity, using the pRL-TK plasmid encoding $\mathrm{C}$ luciferase as a control. Firefly and Renilla luciferase activity was measured using the Dual-Luciferase Reporter Assay System (Promega, Madison, WI, USA).

\subsection{Statistical Analysis}

The results are presented as means \pm standard deviation (SD). Statistical analysis of the groups was performed using Student's t-test or one-way ANOVA with the LSD post-hoc test. Statistical significance was set at ${ }^{*} p<0.05$ and ${ }^{* *} p<0.01$.

Supplementary Materials: The following are available online at https: / www.mdpi.com/article/10 $.3390 /$ biom11050721/s1, Figure S1: The optimal concentration of DEX in PSC cells and quantitative detection of differentially expressed genes in muscle, Figure S2: Prediction of active sites in the $S L 2 A 4$ promoter.

Author Contributions: Conceptualization, J.C., X.-L.D. and W.-J.X.; methodology, X.-L.D., W.-J.X., J.L.S., C.-T.G., K.G., R.Z. and S.-W.J.; validation, X.-L.D. and W.-J.X.; writing-original draft preparation, X.-L.D.; writing-review and editing, X.-L.D. and W.-J.X.; supervision, X.-L.D. All authors have read and agreed to the published version of the manuscript. 
Funding: This research was funded by National Key R\&D Program of China (2017YFD0502000), National Natural Science Foundation of China (31301946, 31402051), The Fundamental Research Funds for the Central Universities (2662020DKPY012), Research Funds for Hubei Key Laboratory of Animal Embryo Engineering and Molecular Breeding (KLAEMB-2019-01) and Key R \& D projects of Hubei Province (2020BBB069).

Institutional Review Board Statement: The study was conducted according to the guidelines of Good Laboratory Practice, and approved by the Institutional Animal Care and Use Committee at Huazhong Agricultural University. (protocol code HZAUSW-2017-001, approved 26 January 2017).

Data Availability Statement: The data presented in this study are available in the article and Supplementary Material.

Acknowledgments: We thank Jiawei Wan, Yuxuan Yang, Zhuxia Zhong and Jian Huang for his help during Isolation of primary skeletal muscle cells.

Conflicts of Interest: The authors declare no conflict of interest.

\section{References}

1. Buckingham, J.C. Glucocorticoids: Exemplars of multi-tasking. Br. J. Pharmacol. 2006, 147 (Suppl. 1), S258-S268. [CrossRef]

2. Burgering, B.M.; Kops, G.J. Cell cycle and death control: Long live Forkheads. Trends Biochem. Sci. 2002, 27, 352-360. [CrossRef]

3. Cen, X.; Liu, S.; Cheng, K. The role of toll-like receptor in inflammation and tumor immunity. Front. Pharmacol. 2018, 9, 878. [CrossRef]

4. Correa-Giannella, M.L.; Machado, U.F. SLC2A4gene: A promising target for pharmacogenomics of insulin resistance. Pharmacogenomics 2013, 14, 847-850. [CrossRef]

5. Feldman, S.; Weidenfeld, J. Electrical stimulation of the dorsal hippocampus caused a long lasting inhibition of ACTH and adrenocortical responses to photic stimuli in freely moving rats. Brain. Res. 2001, 911, 22-26. [CrossRef]

6. Fleming, J.E.; Bensch, K.G. Oxidative stress as a causal factor in differentiation and aging: A unifying hypothesis. Exp. Gerontol. 1991, 26, 511-517. [CrossRef]

7. Gao, L.; Wang, G.; Liu, W.N.; Kinser, H.; Franco, H.L.; Mendelson, C.R. Reciprocal feedback between miR-181a and E2/ERalpha in myometrium enhances inflammation leading to labor. J. Clin. Endocrinol. Metab. 2016, 101, 3646-3656. [CrossRef] [PubMed]

8. Goldberg, A.L.; Tischler, M.; DeMartino, G.; Griffin, G. Hormonal regulation of protein degradation and synthesis in skeletal muscle. Fed. Proc. 1980, 39, 31-36. [PubMed]

9. Goodman, C.A.; Mabrey, D.M.; Frey, J.W.; Miu, M.H.; Schmidt, E.K.; Pierre, P.; Hornberger, T.A. Novel insights into the regulation of skeletal muscle protein synthesis as revealed by a new nonradioactive in vivo technique. FASEB J. 2011, 25, 1028-1039. [CrossRef]

10. Hancock, E.J.; Ang, J.; Papachristodoulou, A.; Stan, G.B. The interplay between feedback and buffering in cellular homeostasis. Cell Syst. 2017, 5, 498-508.e423. [CrossRef] [PubMed]

11. Huang, H.; Regan, K.M.; Lou, Z.; Chen, J.; Tindall, D.J. CDK2-dependent phosphorylation of FOXO1 as an apoptotic response to DNA damage. Science 2006, 314, 294-297. [CrossRef]

12. Jin, B.; Li, Y.P. Curcumin prevents lipopolysaccharide-induced atrogin-1/MAFbx upregulation and muscle mass loss. J. Cell Biochem. 2007, 100, 960-969. [CrossRef] [PubMed]

13. Kadmiel, M.; Cidlowski, J.A. Glucocorticoid receptor signaling in health and disease. Trends Pharmacol. Sci. 2013, 34, 518-530. [CrossRef] [PubMed]

14. Kamei, Y.; Miura, S.; Suzuki, M.; Kai, Y.; Mizukami, J.; Taniguchi, T.; Mochida, K.; Hata, T.; Matsuda, J.; Aburatani, H.; et al. Skeletal muscle FOXO1 (FKHR) transgenic mice have less skeletal muscle mass, down-regulated Type I (slow twitch/red muscle) fiber genes, and impaired glycemic control. J. Biol. Chem. 2004, 279, 41114-41123. [CrossRef]

15. Kino, T.; Manoli, I.; Kelkar, S.; Wang, Y.; Su, Y.A.; Chrousos, G.P. Glucocorticoid receptor (GR) beta has intrinsic, GRalphaindependent transcriptional activity. Biochem. Biophys. Res. Commun. 2009, 381, 671-675. [CrossRef]

16. Klip, A.; McGraw, T.E.; James, D.E. Thirty sweet years of GLUT4. J. Biol. Chem. 2019, 294, 11369-11381. [CrossRef]

17. Lattin, C.R.; Kelly, T.R. Glucocorticoid negative feedback as a potential mediator of trade-offs between reproduction and survival. Gen. Comp. Endocrinol. 2020, 286, 113301. [CrossRef] [PubMed]

18. Lin, H.; Decuypere, E.; Buyse, J. Oxidative stress induced by corticosterone administration in broiler chickens (Gallus gallus domesticus) 2. Short-term effect. Comp. Biochem. Physiol. B Biochem. Mol. Biol. 2004, 139, 745-751. [CrossRef]

19. Lofberg, E.; Gutierrez, A.; Wernerman, J.; Anderstam, B.; Mitch, W.E.; Price, S.R.; Bergstrom, J.; Alvestrand, A. Effects of high doses of glucocorticoids on free amino acids, ribosomes and protein turnover in human muscle. Eur. J. Clin. Investig. 2002, 32, 345-353. [CrossRef]

20. Long, Y.C.; Zierath, J.R. AMP-activated protein kinase signaling in metabolic regulation. J. Clin. Investig. 2006, 116, 1776-1783. [CrossRef]

21. Machado, U.F.; Shimizu, I.; Saito, M. Reduced content and preserved translocation of glucose transporter (GLUT 4) in white adipose tissue of obese mice. Physiol. Behav. 1994, 55, 621-625. [CrossRef] 
22. McCroskery, S.; Thomas, M.; Platt, L.; Hennebry, A.; Nishimura, T.; McLeay, L.; Sharma, M.; Kambadur, R. Improved muscle healing through enhanced regeneration and reduced fibrosis in myostatin-null mice. J. Cell Sci. 2005, 118 Pt 15, 3531-3541. [CrossRef]

23. Motohashi, N.; Asakura, Y.; Asakura, A. Isolation, culture, and transplantation of muscle satellite cells. J. Vis. Exp. 2014, 86, 50846. [CrossRef]

24. Newton, R. Molecular mechanisms of glucocorticoid action: What is important? Thorax 2000, 55, 603-613. [CrossRef]

25. Oakley, R.H.; Webster, J.C.; Jewell, C.M.; Sar, M.; Cidlowski, J.A. Immunocytochemical analysis of the glucocorticoid receptor alpha isoform (GRalpha) using GRalpha-specific antibody. Steroids 1999, 64, 742-751. [CrossRef]

26. Pariante, C.M.; Miller, A.H. Glucocorticoid receptors in major depression: Relevance to pathophysiology and treatment. Biol. Psychiatry 2001, 49, 391-404. [CrossRef]

27. Qi, D.; Rodrigues, B. Glucocorticoids produce whole body insulin resistance with changes in cardiac metabolism. Am. J. Physiol. Endocrinol. Metab. 2007, 292, E654-E667. [CrossRef] [PubMed]

28. Rafacho, A.; Ortsater, H.; Nadal, A.; Quesada, I. Glucocorticoid treatment and endocrine pancreas function: Implications for glucose homeostasis, insulin resistance and diabetes. J. Endocrinol. 2014, 223, R49-R62. [CrossRef] [PubMed]

29. Reul, J.M.; de Kloet, E.R. Two receptor systems for corticosterone in rat brain: Microdistribution and differential occupation. Endocrinology 1985, 117, 2505-2511. [CrossRef]

30. Richter, E.A.; Hargreaves, M. Exercise, GLUT4, and skeletal muscle glucose uptake. Physiol. Rev. 2013, 93, 993-1017. [CrossRef] [PubMed]

31. Saltiel, A.R.; Kahn, C.R. Insulin signalling and the regulation of glucose and lipid metabolism. Nature 2001, 414, 799-806. [CrossRef]

32. Saxton, R.A.; Sabatini, D.M. mTOR signaling in growth, metabolism, and disease. Cell 2017, 169, 361-371. [CrossRef] [PubMed]

33. Shimizu, N.; Yoshikawa, N.; Ito, N.; Maruyama, T.; Suzuki, Y.; Takeda, S.; Nakae, J.; Tagata, Y.; Nishitani, S.; Takehana, K.; et al. Crosstalk between glucocorticoid receptor and nutritional sensor mTOR in skeletal muscle. Cell Metab. 2011, 13,170-182. [CrossRef] [PubMed]

34. Shuai, X.; Tao, K.; Mori, M.; Kanda, T. Bariatric surgery for metabolic syndrome in obesity. Metab. Syndr. Relat. Disord. 2015, 13, 149-160. [CrossRef]

35. Stinckens, A.; Luyten, T.; Bijttebier, J.; Van den Maagdenberg, K.; Dieltiens, D.; Janssens, S.; De Smet, S.; Georges, M.; Buys, N. Characterization of the complete porcine MSTN gene and expression levels in pig breeds differing in muscularity. Anim. Genet. 2008, 39, 586-596. [CrossRef]

36. Strack, A.M.; Sebastian, R.J.; Schwartz, M.W.; Dallman, M.F. Glucocorticoids and insulin: Reciprocal signals for energy balance. Am. J. Physiol. 1995, 268 Pt 2, R142-R149. [CrossRef]

37. Vegiopoulos, A.; Herzig, S. Glucocorticoids, metabolism and metabolic diseases. Mol. Cell Endocrinol. 2007, 275, 43-61. [CrossRef]

38. Wan, X.; Wang, D.; Xiong, Q.; Xiang, H.; Li, H.; Wang, H.; Liu, Z.; Niu, H.; Peng, J.; Jiang, S.; et al. Elucidating a molecular mechanism that the deterioration of porcine meat quality responds to increased cortisol based on transcriptome sequencing. Sci. Rep. 2016, 6, 36589. [CrossRef]

39. Zhang, T.; Wang, F.; Xu, H.X.; Yi, L.; Qin, Y.; Chang, H.; Mi, M.T.; Zhang, Q.Y. Activation of nuclear factor erythroid 2-related factor 2 and PPARgamma plays a role in the genistein-mediated attenuation of oxidative stress-induced endothelial cell injury. Br. J. Nutr. 2013, 109, 223-235. [CrossRef]

40. Zheng, X.; Bi, W.; Yang, G.; Zhao, J.; Wang, J.; Li, X.; Zhou, X. Hyperglycemia induced by chronic restraint stress in mice is associated with nucleus tractus solitarius injury and not just the direct effect of glucocorticoids. Front. Neurosci. 2018, 12, 983. [CrossRef] 